\title{
AN IMMPDAF SOLUTION TO BENCHMARK PROBLEM FOR TRACKING IN CLUTTER AND STANDOFF JAMMER
}

\author{
- Donka ANGELOVA, Emil SEMERDJIEV, Ludmila \\ MIHAYLOVA and Xiao RONG-LI
}

\section{Introduction}

Multiple target tracking is a very important and rapidly developing area. The formulation of a clearly defined standard or benchmark problem for evaluation and comparison of the various existing algorithms is necessary. Researchers have established such a unifying general problem that imposes different and contradictory requirements in the face of the first benchmark problem (BP) defined in ${ }^{4}$ and further extended in ${ }^{6}$. The first benchmark problem considers only aircraft tracking and pointing/ scheduling of a phased array radar. The second benchmark problem ${ }^{6,7}$ involves the presence of False Alarms (FA) and Electronic Countermeasures (ECM) and requires radar resources management. The tracking filter performance criterion is the minimization of a weighted combination of a radar time and energy at the cost of a maximum $4 \%$ tracks' loss.

Previous results devoted to this problem have shown that the Interacting Multiple Model (IMM) filtering algorithm ${ }^{8}$ is the most efficient and cost-effective tool for tracking highly maneuvering targets. ${ }^{3,9,10}$ Additionally the presence of FA and ECM requires sophisticated data association approaches such as Probabilistic Data Association (PDA) or Multiple Hypothesis Tracking. ${ }^{3}$

In the present paper a solution to the benchmark problem based on the combined IMM estimator and PDA technique is proposed. An IMMPDA tracking filter satisfying the benchmark performance criteria is designed. It is realized by using appropriate Extended Kalman Filters (EKF) in the IMM configuration, adaptive scheme for track formation and adaptive radar beam pointing control in order to maximize the revisit interval. 
The complete solution to the BP requires the development of the neutralizing techniques for ECM, in particular against a Standoff Jammer (SOJ). The IMMPDA filtering approach has been naturally extended in ${ }^{11}$ to accomplish this task. When the jammer influence is taken into account, the detection threshold and the radar waveform are adaptively selected to ensure a constant false alarm rate and a predetermined target detection probability. This methodology is implemented in the work.

The paper is organized as follows. Section 2 concisely summarizes the idea of the IMMPDA filtering approach to hybrid system estimation. In Section 3, the concrete implementation of the IMMPDA filter for BP solution is described. The SOJ neutralizing technique is briefly described in Section 4 and the simulation results are given in Section 5.

\section{IMMPDA Filtering of Hybrid Systems}

The behavior of a maneuvering target can be adequately described in the terminology of the stochastic hybrid systems. The base state vector $x(k) \in R^{n_{x}}$ of the discrete hybrid system

$$
\begin{aligned}
& x(k)=f(M(k), x(k-1))+G[M(k)] v(k-1, M(k)) \\
& z(k)=h(M(k), x(k))+w(k, M(k)), k=1,2, \ldots
\end{aligned}
$$

is estimated, where $z(k) \in R^{n_{z}}$ is the measurement vector, $v(k) \in R^{n_{x}}$ and $w(k) \in R^{n_{z}}$ are respectively the system and measurement noises, assumed to be white and mutually uncorrelated, with zero means and variances, respectively $Q(k)$ and $R(k)$. The system (1)-(2) at time $k$ is among $r$ possible modal states (models), depending on the parameter $M(k) \in\{1,2, \cdots, r\}$, where $M(k)=i$ denotes that the $i$-th system mode is in effect during the sampling interval $T$ ending at time $k$. The mode sequence $\{M(k)\}_{k=1,2, \ldots}$ is assumed to be a Markov chain with known initial mode probabilities $\mu_{i}=P\{M(0)=i\}$ and transitional probabilities $p_{i j}=P\{M(k)=j / M(k-1)=i\}, \quad i, j \in M$, where $P\{$.$\} is the notation for$ probability .

In the presence of clutter several measurements are received from the sensor at time k, i.e. $Z(k)=\left\{z_{i}(k)\right\}_{i=1}^{m(k)}$. The aim of the hybrid estimation is to provide the system state and modal state estimates on the basis of the cumulative set of measurements $Z^{k}=\{Z(k)\}_{k=1}^{k}$. In general, suboptimal Bayesian procedures are applied and the 
final estimate is a weighted sum of the estimates generated by $r$ working in parallel Kalman filters. In the absence of model uncertainty, the single model minimum variance estimate of the state is computed by the PDA filter ${ }^{1}$ :

$$
\hat{x}(k / k)=\sum_{i=0}^{m(k)} E\left[x(k) \mid \theta_{i}(k), Z^{k}\right] P\left\{\theta_{i}(k), Z^{k}\right\}
$$

where $\theta_{i}(k)$ is the event that $z_{i}(k)$ is the correct measurement from the target at $k$ and $\theta_{0}(k)$ - the event that none of the measurements is correct. $E\{$.$\} is the$ mathematical expectation operator. When both model uncertainty and measurement origin uncertainty are present, the state estimate is given within the framework of the IMM filtering approach by the total probability theorem:

$$
\begin{aligned}
& \hat{x}(k / k)=\sum_{j=1}^{r}\left\{\sum_{i=0}^{m(k)} E\left[x(k) \mid \theta_{i}(k), M_{j}, Z^{k}\right] P\left\{\theta_{i}(k) \mid M_{j}, Z^{k}\right\}\right\} P\left\{M_{j} \mid Z^{k}\right\} \\
& \text { or } \quad \hat{x}(k / k)=\sum_{j=1}^{r} \hat{x}^{j}(k / k) \mu_{j}(k)
\end{aligned}
$$

where $\hat{x}^{j}(k / k)$ is the output of the $j$-th PDA filter based on the $j$-th model and $\mu_{j}(k)=P\left\{M_{j} \mid Z^{k}\right\}, j=1, \ldots, r \quad$ is the conditional posterior probability of mode $j$. The associated with the estimate error covariance $P(k / k)$ takes into account the effect of the model and measurement origin uncertainties.

\section{IMMPDA Filter for BP Solution}

A number of mutually connected tasks for precise target tracking are posed in the benchmark formulation. ${ }^{6}$ Their optimum solution minimizes both the radar time and energy. The tracking algorithm involves track formation and maintenance, as well as the choice of target revisit interval. One solution to these tracking problems is presented here.

The IMMPDA filter is an algorithm for tracking that can realize simultaneously track formation and maintenance. It provides a quantitative assessments for track termination and tracking capability in clutter. In the present work IMMPDA filter is implemented only for track maintenance. The track termination is determined here according to the criterion for lost tracks suggested in ${ }^{7}$. A simplified version of track formation is accomplished in view of the specific benchmark problem features. As a result the computational load and the radar energy are reduced. 


\subsection{Track formation}

The track formation is a difficult task in the presence of FA. On the basis of the sequence of measurements with a high signal-to-noise ratio (SNR), a track is formed by the Least-Squares (LS) method. This technique provides the initial target state estimates and the associated covariance matrix. The estimation accuracy, however, greatly depends on the target ranges which vary from 20 to $100 \mathrm{~km}$ in the considered benchmark trajectories. For this reason the number of the measurements and sampling intervals in the LS procedure are determined according to the measured range to the target. In addition, the highest energy waveform is used for the remote fast targets.

\subsection{Track maintenance}

The suitable choice of motion models, covering well the whole range of target flight modes, is the first important task in the IMMPDA filter design. The hardest target maneuvers require lateral accelerations up to $7 g$ and longitudinal accelerations up to $2 \mathrm{~g}$. The targets maneuver mainly through turns with the highest intensity $(7 \mathrm{~g})$. That is why it is proposed here a nonlinear approximate turn model to be used for the maneuvering segments. ${ }^{2}$ As the angular rate of the turns is not known and varies in a wide range, it is included in the state vector and is estimated by the filter.

The following set of models is suggested for the IMM track maintenance algorithm:

a) No target model $\left(M_{1}\right)$ takes into account an undetectable or "false" target. It is usually selected as a second order $\operatorname{model}^{1}$ with low noise level, corresponding to the uniform motion, with a target detection probability $P_{D}=0$. Its posterior mode probability can be used as a criterion for track termination. According to (1), $f[M(k), x(k-1)]=f\left[M_{1}, x(k-1)\right]=F x$, where the state space vector $x=[x, \dot{x}, y, \dot{y}, z, \dot{z}]^{T}$ contains the target positions and velocities in a Cartesian coordinate frame and the matrix $F$ has the form described in ${ }^{1 \text { ( pp. 228). }}$

b) A second order model ( $\left.M_{2}\right)$ considers the nearly constant velocity $(\mathrm{CV})$ motion of a nonmaneuvering target. It is usually selected with low noise level and $P_{D} \neq 0$, given by the target's expected SNR. For the state space vector $x=[x, \dot{x}, y, \dot{y}, z, \dot{z}]^{T}$, the CV target motion model is linear. According to (1), $f[M(k), x(k-1)]=f\left[M_{2}, x(k-1)\right]=F x$, where the matrix $F$ is the same as in a).

c) A maneuver model $\left(M_{3}\right),\left(P_{D} \neq 0\right)$ takes into account the on-going maneuvers. It is a nonlinear coordinated turn model ${ }^{2}$ with unknown angular rate $\omega$, incorporated into the state vector $x=[x, \dot{x}, y, \dot{y}, z, \dot{z}, \omega]^{T}$. According to the eq. (1) $f\left[M_{3}, x(k-1)\right]=f(x)$, where 


$$
\begin{aligned}
& f(x)=\left[\begin{array}{llll}
f_{1}^{T}(x) & f_{2}^{T}(y) & f_{3}^{T}(z) & f_{4}^{T}(\omega)
\end{array}\right]^{T}, \\
& f_{1}(x)=\left[x+T \dot{x}-\frac{T^{2}}{2} \dot{y} \omega, \dot{x}-T \dot{y} \omega-\frac{T^{2}}{2} \dot{x} \omega^{2}\right] \\
& f_{2}(y)=\left[y+T \dot{y}+\frac{T^{2}}{2} \dot{x} \omega, \dot{y}+T \dot{x} \omega-\frac{T^{2}}{2} \dot{y} \omega^{2}\right] \\
& f_{3}(z)=[z+T \dot{z}, \dot{z}] \\
& f_{4}(\omega)=\omega \text {. }
\end{aligned}
$$

d) A maneuver start / termination model $\left(M_{4}\right)\left(P_{D} \neq 0\right)$ for transitional flight segments (between constant speed and turns), necessary for tracking highly maneuvering targets. It is selected as a second order model with a high level of noise: $f[M(k), x(k-1)]=f\left[M_{4}, x(k-1)\right]=F x$, where $x=[x, \dot{x}, y, \dot{y}, z, \dot{z}]^{T}$ and the transition matrix $F$ is the same as in the models a) and b).

The second task of the IMMPDA filter design comprises the selection of prior parameters: the process noise variance and the Markovian transition matrix. In view of the dynamics of the simulated in the BP targets, the standard deviations of the process noise components for the four models are chosen as:

$$
M_{1}: \quad \sigma_{v_{1}}=2.8 \mathrm{~m} / \mathrm{sec}^{2} ; \quad M_{2}: \quad \sigma_{v_{2}}=2.8 \mathrm{~m} / \mathrm{sec}^{2}
$$

$$
M_{3}:\left\{\begin{array}{c}
\sigma_{v_{3} x}=\max \{10, \min [a b s(-\dot{y} \omega), 70]\} \mathrm{m} / \mathrm{sec}^{2} \\
\sigma_{v_{3} y}=\max \{10, \min [a b s(\dot{x} \omega), 70]\} \mathrm{m} / \mathrm{sec}^{2} \\
\sigma_{v_{3} z}=15 \mathrm{~m} / \mathrm{sec}^{2} \\
\sigma_{v_{3} \omega}=0.064 \mathrm{rad} / \mathrm{sec}
\end{array}\right.
$$

$$
M_{4}: \quad \sigma_{v_{4}}=\min \{50 T, 70\} \mathrm{m} / \mathrm{sec}^{2},
$$


where $\ddot{x}=-\dot{y} \omega$ and $\ddot{y}=\dot{x} \omega$ are the accelerations in the "coordinated turn" model. The matrix $G[M(k)]=G$ from (1) has the usual form ${ }^{1}$ in the four models.

The elements of the transition matrix can be chosen as follows ${ }^{2}$ :

$$
p_{i j}(T)=\left\{\begin{array}{llr}
\exp \left(-T / \tau_{i}\right) & \text { if } & j=i \\
p_{i j}\left[1-p_{i i}(T)\right] & \text { if } & j \neq i
\end{array}\right.
$$

where $\tau_{i}$ is the expected sojourn time of the $i$-th mode. In the present IMM implementation, however, the following constant values are assigned to the transition probabilities in order to reduce the computational time:

$$
p=\left[\begin{array}{llll}
0.94 & 0.02 & 0.02 & 0.02 \\
0.02 & 0.83 & 0.13 & 0.02 \\
0.02 & 0.09 & 0.86 & 0.03 \\
0.02 & 0.08 & 0.20 & 0.70
\end{array}\right] .
$$

The initial mode probabilities are set to : $p_{1}^{0}=0.1 ; p_{2}^{0}=p_{3}^{0}=p_{4}^{0}=0.3$;

\subsection{Measurement model}

Since the radar measurements are received in a spherical coordinate system, the measurement vector $z$ comprises the range $r$, the bearing $b$ and elevation $e$ angles, i.e. $z=\left(\begin{array}{lll}r & b & e\end{array}\right)^{T}$. The measurement equation (2) has the form:

$$
h(x)=\left[\sqrt{x^{2}+y^{2}+z^{2}}, \quad \tan ^{-1} \frac{y}{x}, \tan ^{-1} \frac{z}{\sqrt{x^{2}+y^{2}}}\right]^{T} .
$$

The nonlinearity in the relationships $f(x)$ and $h(x)$ imposes the Extended Kalman Filters application in the IMM configuration.

\subsection{Adaptive sampling}

An adaptive computation of the sampling interval is needed when the radar resources have to be saved. It is achieved by using a short sampling interval during maneuvers and a long one during nonmaneuvering trajectory segments. Here, the sampling interval selection scheme, suggested in ${ }^{11}$, is adopted:

- $\quad$ a set $\Delta$ of fixed sampling intervals $T$ is determined; 
- for the largest $T$, the predicted positions and innovation covariances from the IMM filters are combined, by using IMM predicted mode probabilities;

- the combined innovation standard deviations $\sigma_{k}^{b}, \sigma_{k}^{e}$ in bearing and elevation are compared with the antenna beamwidth in bearing $B_{k}^{b}$ and elevation $B_{k}^{e}$, respectively:

$\sigma_{k}^{b} \leq B_{k}^{b} / K_{b}$ and $\sigma_{k}^{e} \leq B_{k}^{e} / K_{e}$,

where $K_{b}$ and $K_{e}$ are threshold parameters;

- if any of these angle deviations exceeds the threshold, the test is repeated for the next shorter $T$;

- if no measurements are received, the sampling interval $T$ is assumed to be equal to $0.1 \mathrm{sec}$.

In our implementation the following set of sampling intervals is accepted:

$$
\Delta=\{0.1,0.5,0.9,1.3,1.7,2.1,2.5,2.9,3.3,3.7\}
$$

At first the threshold $K_{b}=K_{e}=K$ is selected equal to $K=4.2$. If the target is not detected, the threshold $K$ is augmented to the value of 6 . During the next subsequent scans, the sampling interval increases to its maximum value $T=3.7 \mathrm{sec}$, (according to the described above logic), and then $K$ is returned to its ordinary base value of 4.5 .

\section{Neutralizing the SOJ}

The neutralizing technique for The SOJ, presented in $^{11}$, is realized in the paper. The SOJ motion parameters are estimated by EKF based on angles only measurements, received at the radar in passive mode. The jammer tracker using azimuth and elevation angles, their derivatives and a $2.0 \mathrm{sec}$ update rate is implemented ${ }^{7}$ to predict the jammer position. The predicted estimate of the jammer power level is used for an adaptive selection of the detection threshold in order to maintain a constant false alarm rate. To maintain the predetermined target detection probability, the radar waveform is also adaptively selected by an additional assessment of the target radar cross section. ${ }^{11}$ 


\section{Simulation results}

The algorithm performance is evaluated over six standard BP test scenarios. ${ }^{7}$ The well known criteria for filter performance are used: the energy and radar time costs, position and velocity root-mean-square errors (RMSE), computational requirements, percentage of lost tracks. ${ }^{7}$

The number of tracks lost is a key performance indicator for a filter, operating in a cluttered environment and ECM. The main measures of performance, concerning the energy and radar time costs have the form ${ }^{7}$ :

$$
C_{i}=\bar{E}_{\text {ave }}+\alpha_{i} \bar{T}_{\text {ave }}, \quad i=1,2 ; \quad \alpha_{1}=10^{3} ; \quad \alpha_{2}=10^{5},
$$

where $\bar{E}_{\text {ave }}$ is the average radar energy per second, $\bar{T}_{\text {ave }}$ is the average radar time per second and $\alpha_{i}$ is a given weighting parameter.

The results obtained for 200 Monte Carlo runs in the presence of FA and SOJ are shown in Table 1 . The average values of the parameters $C_{1}$ and $C_{2}$, computed over the six scenarios by taking into account the respective parameters of target 1 two times (as is required in ${ }^{7}$ ), are given in the last row of Table 1. It can be seen from the results that the realized IMMPDA algorithm version satisfies the BP requirements for all six target scenarios.

Figures 1 through 6 illustrate the results obtained for the most difficult scenario 6 . The waveform adaptation, corresponding to the selected detection threshold can be seen in Figure 1. The waveform peaks follow the changes in the acceleration magnitude and the SOJ influence. The sampling interval (Figure 2) is larger during nonmaneuvering phases of motion $(\approx 3 \div 3.6 \mathrm{sec})$ in comparison to the maneuvering periods of flight $(\approx 1.5 \mathrm{sec})$. Therefore, the IMM innovation standard deviations give a good measure for the confidence of the predicted state estimates. The evolution of the IMM mode probabilities for one run is presented in Figure 5. The posterior mode probabilities correctly identify the true system mode for all target scenarios. The rapid response to the changes in the target behavior ensures acceptable RMS Errors. The average position and velocity RMSE are shown in Figures 3 and 4, respectively. The peak RMS position errors do not exceed $500 \mathrm{~m}$; the top velocity RMSE are of the order of $250 \mathrm{~m} / \mathrm{s}$. The average estimated value of the angular rate, which is a state component of the maneuvering model, is presented in Figure 6. It is obvious from the simulation results that the performance of the proposed tracking algorithm is comparable to the performance of the algorithm derived in ${ }^{11}$. The average sampling interval $(2.85 \mathrm{~s})$ and the average power $(8.24 \mathrm{~W})$ are approximately the same as the respective parameters $(2.71 \mathrm{~s}$ and $8.6 \mathrm{~W})$ in ${ }^{11}$. 
Table 1: IMMPDAF performance in the presence of FA and SOJ

\begin{tabular}{|c|c|c|c|c|c|c|c|}
\hline Target & $\begin{array}{c}\text { Sample } \\
\text { Period } \\
(\mathbf{s})\end{array}$ & $\begin{array}{c}\text { Ave. } \\
\text { Power (w) }\end{array}$ & $\begin{array}{c}\text { Pos. } \\
\text { RMSE } \\
(\mathbf{m})\end{array}$ & $\begin{array}{c}\text { Vel. } \\
\text { RMSE } \\
(\mathbf{m} / \mathbf{s})\end{array}$ & $\begin{array}{c}\text { Cost } \\
C_{1}\end{array}$ & $C_{2}$ & $\begin{array}{c}\text { Cost } \\
\text { Tracks }\end{array}$ \\
\hline 1 & 2.91 & 7.28 & 115.0 & 50.27 & 7.63 & 41.65 & 0 \\
\hline 2 & 2.88 & 6.16 & 100.3 & 52.18 & 6.51 & 40.84 & 0 \\
\hline 3 & 2.87 & 10.36 & 148.7 & 79.15 & 10.71 & 45.18 & 0 \\
\hline 4 & 2.91 & 3.07 & 45.81 & 36.55 & 3.42 & 37.37 & 0 \\
\hline 5 & 2.77 & 15.91 & 171.4 & 74.49 & 16.27 & 51.94 & 0 \\
\hline 6 & 2.71 & 7.62 & 114.8 & 72.44 & 7.99 & 44.48 & 1 \\
\hline Ave. & 2.85 & 8.24 & - & - & 8.60 & 43.31 & - \\
\hline
\end{tabular}

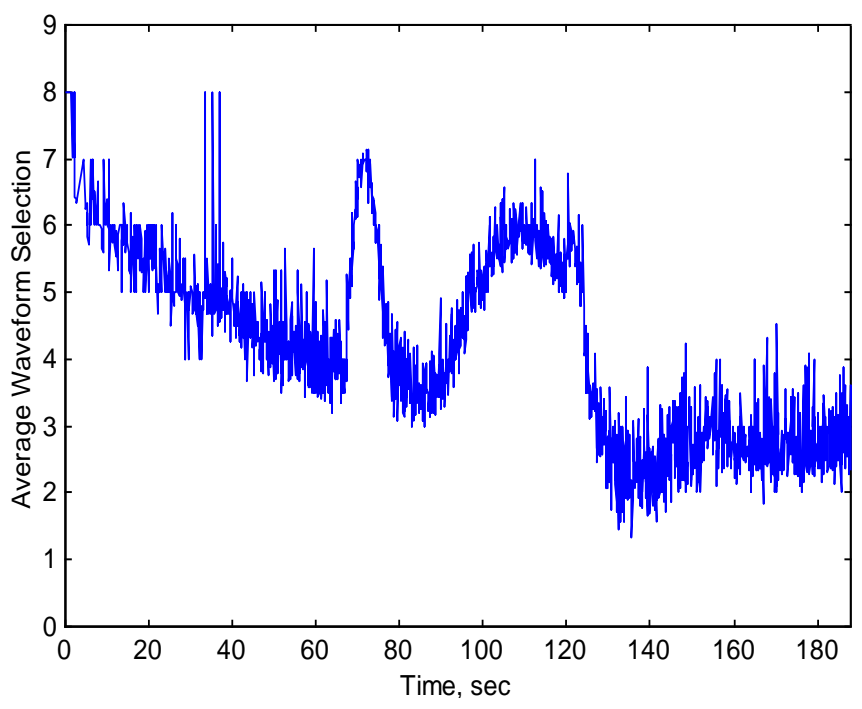

Figure 1 


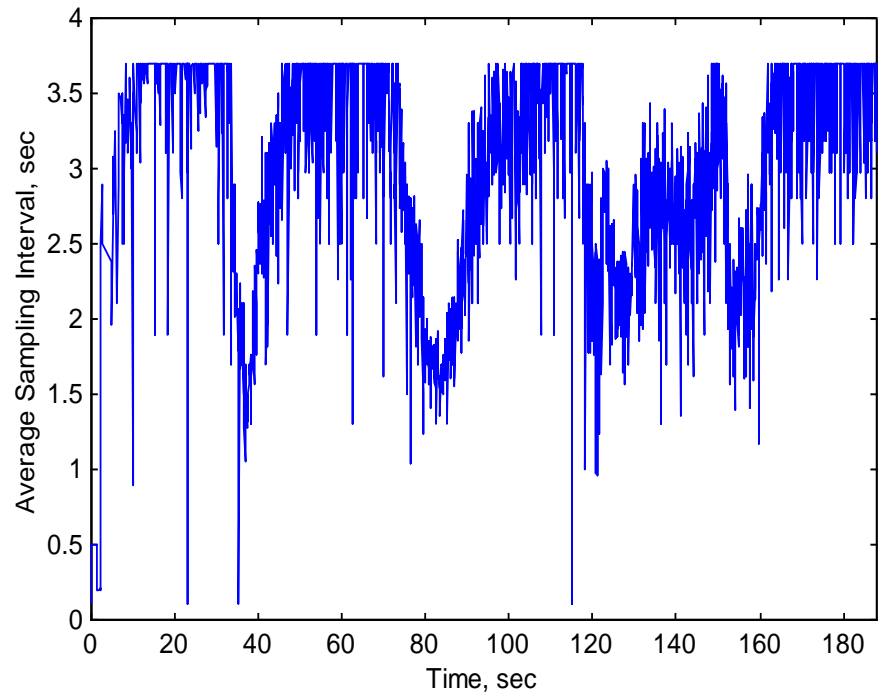

Figure 2

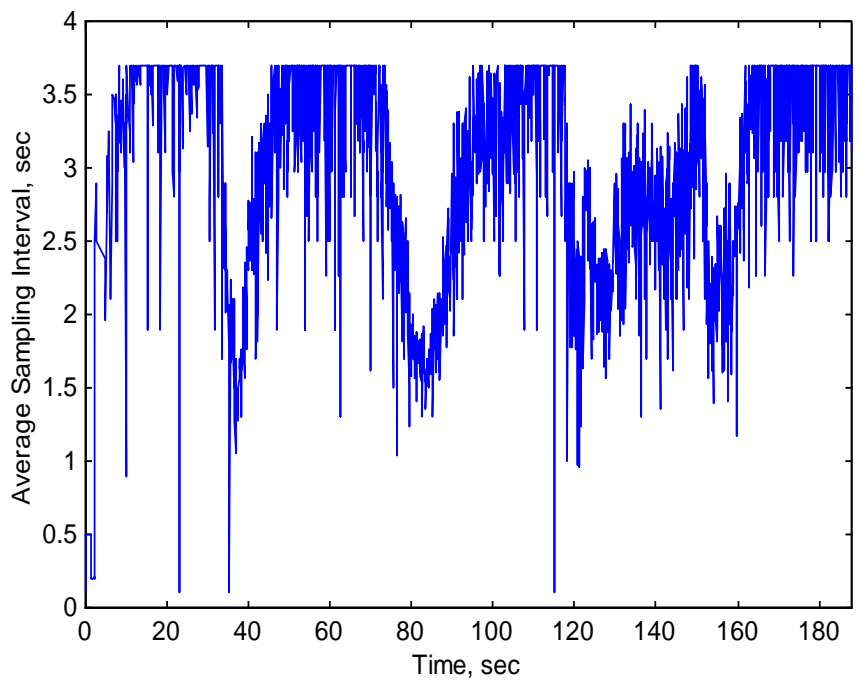

Figure 3 


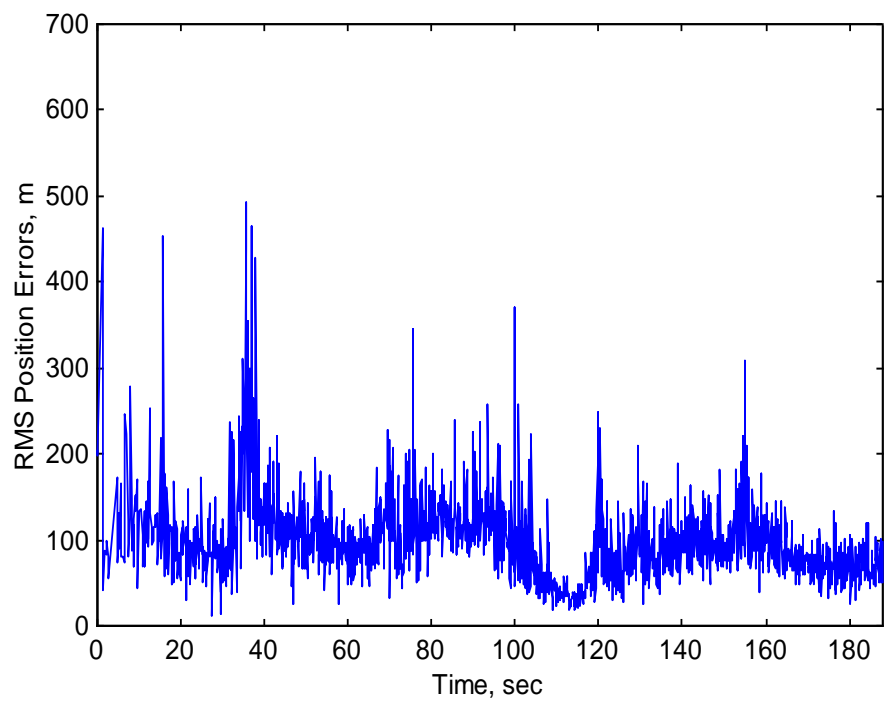

Figure 4

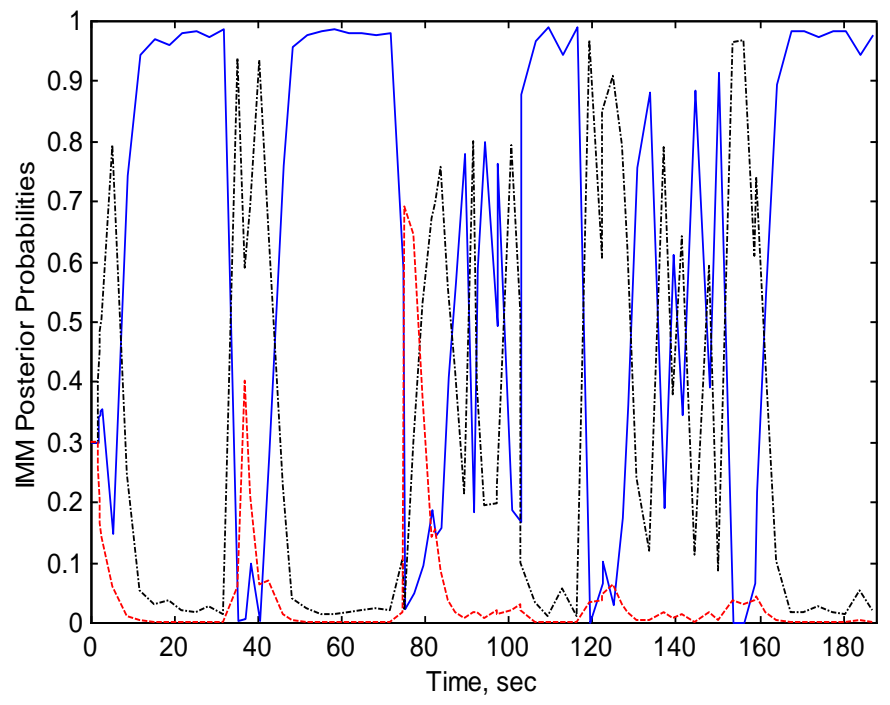

Figure 5 


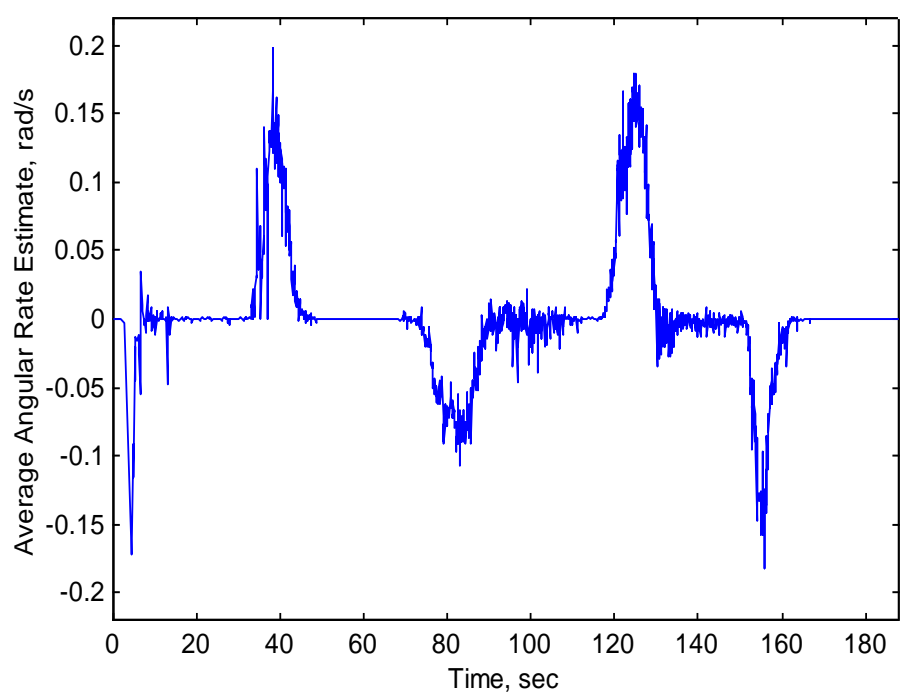

Figure 6

\section{Conclusions}

Preliminary results of an ongoing study are reported in the paper. An algorithm is proposed for radar management and tracking of maneuvering aircraft in the presence of clutter and Standoff Jammer. It is based on the advanced IMMPDA filtering approach for hybrid system estimation. The performance of the designed algorithm is evaluated by Monte Carlo simulations. Results obtained over six standard benchmark test scenarios are given. They show that the tracking filter characteristics satisfy the benchmark restrictions and they are close to the performance of the algorithms, recently published in the literature.

The further investigation comprises:

- implementation of the idea of the optimal initialization, described in ${ }^{12}$;

- replacement of the PDAF with IPDAF for track formation, confirmation and termination;

- replacement of the PDAF with Probabilistic Strongest Neighbor Filter or Interacting 2-model $\mathrm{PDAF}^{5}$;

- implementation of the Decomposition and Fusion Method for handling Range Gate Pull-Off ECM. 


\section{Acknowledgement}

The work of DA, ES and LM in this study was partially supported by Bulgarian National Science Fund grant \# I-808/98. X.R.-L. received support through ONR Grant N00014-97-1-0570, NSF Grant ECS-9734285, and LEQSF Grant (1996-99)RD-A-32.

\section{References}

1. Y. Bar-Shalom and X. Rong-Li, Multitarget-Multisensor Tracking: Principles and Techniques (Storrs, CT: YBS Publishing, 1995).

2. Y. Bar-Shalom, ed., Multitarget-Multisensor Tracking: Applications and Advances, vol. II (Artech House, 1992).

3. S. Blackman, M. Bush, and R. Popoli, "IMM/MHT tracking and data association for benchmark problem," In Proceedings of the 1995 American Control Conference (1995), 2606-2610.

4. W. Blair, G. Watson and S. Hoffman, "Benchmark problem for beam pointing control of phased array radar against maneuvering targets," Proceedings of the 1994 American Control Conference (1994), 2071-2075.

5. X. Rong-Li and C. He, "2M-PDAF: An integrated two model probabilistic data association filter," SPIE Conference on Signal and Data Processing of Small Targets (1999).

6. W. Blair, G. Watson, G. Gentry and S. Hoffman, "Benchmark problem for beam pointing control of phased array radar against maneuvering targets in the presence of false alarms and ECM," Proceedings of the 1995 American Control Conference (1995), 2601-2605.

7. W. Blair and G. Watson, "Benchmark for radar resource allocation and tracking targets in the presence of ECM," IEEE Trans. on AES 34, 4 (1998), 1097-1114.

8. H. Blom and Y. Bar-Shalom, "The interacting multiple model algorithm for systems with Markovian switching coefficients," IEEE Trans. on AC 33, 8 (1988), 780-783.

9. E. Daeipour, Y. Bar-Shalom and X. Rong Li, "Adaptive beam pointing control of a phased array radar using an IMM estimator," Proceedings of 1994 American Control Conference (1994), 2093-2097. 
10. V. Jilkov, L. Mihaylova and X.Rong-Li, "An Alternative IMM Solution to Benchmark Tracking Problem,” Proc. of the FUSION'98 (Las Vegas, Nevada: July 6-9, 1998), Vol.2, 924-929.

11. T. Kirubarajan, Y. Bar-Shalom, W. Blair and G. Watson, "IMMPDA solution to benchmark problem for radar resource allocation and tracking in the presence of ECM," IEEE Trans. on AES 34, 4 (1998), 1115-1134.

12. X.Rong-Li and C. He, "Optimal initialization of linear recursive filters," Proc. 37th IEEE Conference on Decision and Control (Tampa, FL: December 1998), 2335-2340.

DONKA STANCHEVA ANGELOVA is assistant research professor at the Central Laboratory for Parallel Processing, Bulgarian Academy of Sciences. She received M.S. degree in Sofia Technical University in 1972 and Ph.D degree in the Bulgarian Army War College in 1990. She is member of ISIF. E-mail: donka@bas.bg.

EMIL ATANASOV SEMERDJIEV is professor at the Central Laboratory for Parallel Processing (CLPP), Bulgarian Academy of Sciences, and leader of the SIGNAL Laboratory at CLPPI. He received D.Sc. degree in Rakovsky War College, Sofia, Bulgaria, in 1990, Ph.D. and M.Sc. in Zhukovsky Air Force Engineering Academy, Moscow, Russia, 1978. He is member of IEEE, AFCEA, ISIF and International Academy for Information Processing and Technologies, Moscow, Russia. Office Address: CLPP-BAS, Acad. G.Bonchev Str., bl.25 A, 1113 Sofia, Bulgaria; Phone: (+359 2) 731 498, Fax: (+359 2) 707 273; E-mail: signal@bas.bg.

LUDMILA STOYANOVA MIHAYLOVA is assistant research professor at the Central Laboratory for Parallel Processing, Bulgarian Academy of Sciences. She received M.S. degree in Sofia Technical University, Bulgaria, in 1989. She specialized in Informatics and Applied Mathematics in 1991, and received Ph.D. degree in Sofia Technical University, in 1996. IEEE, WSES and ISIF member. E-mail: lsm@bas.bg.

XIAO RONG LI is a Professor in the department of Electrical Engineering, University of New Orleans, LA, US. He received the B.S. and M.S. Degrees from Zhejiang University, Hangzhou, Zjeijiang, P.R.C. in 1982 and 1984, respectively, and the M.S. and Ph.D. degrees from the University of Connecticut, Storrs, in 1990 and 1992, respectively, all in Electrical Engineering. Dr. Li has published numerous refereed journal articles, three book chapters, and co-authored (with Y.Bar-Shalom) four books. He has also won several outstanding papers awards. He is an editor for Tracking and Navigation of the IEEE Transaction on Aerospace and Electronic Systems. 